\title{
Reply to: 464_2009_331: Re: A simplified laparoscopic ventral hernia repair: the scroll technique (2008: 22(11) 2527-2531)
}

\author{
Alan A. Saber · Mohamed H. Elgamal
}

Published online: 5 March 2009

(C) Springer Science+Business Media, LLC 2009

We thank Hussain et al. for their interest in our article, "A simplified laparoscopic ventral hernia repair: the scroll technique" [1]. Regarding our case of mesh infection, this patient has a history of skin graft to cover exposed bowel. He developed an incisional hernia that was repaired laparoscopically with ePTFE mesh. Postoperatively, the patient developed necrosis of the skin graft with mesh exposure. Long course of antibiotic and wound care failed to salvage the mesh. We and others agree that in the vast majority of cases removal of exposed (infected) mesh is necessary, particularly if the prosthesis contains a component of ePTFE [2]. The patient underwent mesh removal and wound care and delayed repair of the hernia with biological mesh.

We looked at port site hernia during the follow-up period; however, we did not have any case incisional hernia at $12-\mathrm{mm}$ trocar site. This is attributed to the fact that the open Hasson is a muscle splitting and we routinely close all 10-mm and larger port sites.

It is our observation that the incidence of palpable seroma is high with PTFE mesh. This may be attributed to the poor tissue ingrowths of the ePTFE to the abdominal wall. Susmallian et al. found that the incidence of seroma after laparoscopic repair of hernia with PTFE patch was $100 \%$ on ultrasonic studies [3]. In an effort to diminish the seroma formation, we and others place an abdominal binder, for 1 month, while the patient is still on the operating table. This seems to decrease both the size and duration of the seromas that are clinically significant by as much as 50\% [4].

A. A. Saber $(\bowtie) \cdot$ M. H. Elgamal

Minimally Invasive Surgery, Michigan State University/KCMS,

Kalamazoo, USA

e-mail: saber@kcms.msu.edu
Hussain et al. have described the introduction of the mesh through a trocar, which is placed in the center of the hernia defect itself [5]. Although this technique simplifies the procedure, it also creates some concerns. The proximity of the trocar incision to the mesh may facilitate extension of this trocar site infection to the mesh. In addition, the proximity of the hernia sac (the site of seroma formation) and the central port may facilitate leak of seroma fluid through the central port incision.

\section{References}

1. Saber AA, Elgamal MH, Rao EA, Itawi E, Mancl T (2008) A simplified laparoscopic ventral hernia repair: the scroll technique. Surg Endosc 22:2527-2531

2. LeBlanc KA, Whitaker JM, Bellanger DE, Rhynes VK (2003) Laparoscopic incisional and ventral hernioplasty: lessons learned from 200 patients. Hernia 7:118-124

3. Susmallian S, Gewurtz G, Ezri T, Charuzi I (2001) Seroma after laparoscopic repair of hernia with PTFE patch: is it really a complication? Hernia 5:139-141

4. Chowbey PK, Sharma A, Khullar R, Baijal M, Vashistha A (2000) Laparoscopic ventral hernia repair. J Laparoendosc Adv Surg Tech A $10: 79-84$

5. Hussain A, Mahmood H, Shuaib S, El-Hasani S (2008) Prevention of trocar site incisional hernia following laparoscopic ventral hernia repair. JSLS 12:206-209 